\title{
电子废弃物中金元素的绿色溶解与提取
}

吴松, 张朝*, 龙明昊, 刘永杰

贵州大学化学与化工学院, 贵阳 550025

摘要: 电子废弃物中含有 $\mathrm{Au} 、 \mathrm{Pt} 、 \mathrm{Pd} 、 \mathrm{Ag}$ 等多种贵重金属, 其处理处置的无害化、资源化成为目前一项重要课 题。本文通过使用安全的试剂来溶解金, 从而达到从含金废弃物中回收金的目的, 说明利用配体与金形成配合物 从而有效降低溶金电极电势的化学原理。普及科学原理, 树立科学理念。介绍电子废弃物处置的常识, 普及绿色 化学的观念, 避免新的污染与危险, 树立环境保护的观念。

关键词: 金; 电子废弃物; 绿色化学; 配位化合物

中图分类号: G64; O6

\section{A Green Chemistry Technique for Dissolution and Recovery of Gold from Electronic Wastes}

Song Wu, Chao Zhang ", Minghao Long, Yongjie Liu

College of Chemistry and Chemical Engineering, GuiZhou University, Guiyang 550025, P. R. China.

Abstract: Electronic wastes are a rich source of precious metals, e.g., gold ( $\mathrm{Au})$, platinum (Pt), palladium (Pd) and silver (Ag). The recycling of these metals has significant environmental importance. This article demonstrated a chemical approach for recycling gold-containing wastes by dissolving gold with safe chemicals. In this reaction, a ligand and gold could form a coordination complex and thus decrease the electrode potential for gold dissolution. The aims of this article are to show the chemistry of chemical reactions using the dissolution of gold as an example, the presence and selection of chemical approaches for tackling some of the increasing environmental problems, the importance of our living environment and problems that we need to tackle with more efforts.

Key Words: Gold; Electronic wastes; Green chemistry; Coordination complex

\section{1 引言}

电子废弃物是指废弃的电子与电器设备(Waste electrical and electronic equipment, 简称 WEEE)。 目前电子废弃物的日益增多, 是当今全社会范围日益突出的问题, 一方面污染随处可见, 另一方面 这些废弃物中多种宝贵资源的浪费显而易见。在当今资源日益紧缺的背景下，从废弃物中回收贵金 属(金 $\mathrm{Au}$ 、铂 $\mathrm{Pt}$ 、钯 $\mathrm{Pd}$ 等)资源是一个重大课题, 也引起了环保工作者的广泛关注 ${ }^{[1,2]}$ 。电子废弃物 中的金元素含量较高, 据瑞典 Ronnskar 冶炼厂对个人计算机电路板中的元素分析, 金的质量浓度高 达 $80 \mathrm{~g} \cdot \mathrm{t}^{-1}$, 高于一般金矿石的品位 ${ }^{[3]}$ 。黄金等贵金属由于特殊的化学性质, 氧化溶解一直是广泛的 难题, 并且目前使用方法多数对环境有不良影响, 形成二次污染。以更加清洁、有效的方法来提取 电子废弃物中的金元素是本实验的主要内容。目前全球生产的大约 $70 \%$ 的电子产品最终变成垃圾并 
流向中国, 中国已经成为世界最大的电子 “垃圾场” [4,5]。对我国而言, 形势异常严峻, 面对我国各 地众多盲目生产、缺乏科学指导而产生的二次污染与安全隐患, 有必要对利用配合物溶金的化学原 理进行科普, 普及正确的观念, 避免危险与危害。

金元素以超强的化学稳定性著称, 自然条件下无法氧化并溶解黄金, 在化学手段下, 以氰化物、 王水等试剂进行相关反应, 才可以达到溶解、提取的目的。但众所周知, 氰化物有剧毒, $0.1-0.3 \mathrm{~g}$ 即 可导致人死亡, 而生产过程中如果与酸作用, 产生氰化氢, $\mathrm{HCN}$ 在空气中质量浓度为 $20 \mu \mathrm{g} \cdot \mathrm{mL}^{-1}$ 时, 经过数小时人就产生中毒症状、致死, 在安全上的弱点是显而易见的; 而王水法, 尽管对金的溶解 是有效的, 但过程中由于强腐蚀性, 不仅对设备有苛刻的要求, 给安全也带来隐患, 同时对废弃物 中几乎所有金属都可溶解，造成后期还原提纯的困难，导致回收率低等问题。

面对很高的氧化电极电势, 如何氧化并溶解金元素, 一直是一个难题。我们从溶金的化学原理 入手, 对传统的溶金方案进行梳理, 氭化法、王水法等都是利用了 $\mathrm{CN}^{-} 、 \mathrm{Cl}^{-}$等配体能与金形成配合 物, 并有效降低金的电极电势, 从而实现了对金的溶解。选择无害的试剂, 能最大程度地降低实验 过程的危害, 并实现对资源的再生。本实验采用公众熟知并可食用的如氯化钠、醋酸、维生素 $\mathrm{C}$ 等 试剂入手, 使用安全试剂, 巧妙地利用 $\mathrm{Cl}^{-} 、 \mathrm{I}^{-}$作为配体, 避免了氧化物、王水、录、澳等危险与危 害试剂, 过程更安全, 完成了对电子废弃物中金元素的提取。

本实验一方面可作为本科教学实验, 使学生进一步学习配合物的性质, 认识配合物降低氧化电 极电势的原理; 另一方面可向公众展示, 引导正确科学理念, 避免危险与危害的发生, 并普及电子 垃圾回收的基本知识。

\section{2 实验部分}

\section{1 实验原理}

1) 金元素有着很强的化学稳定性, 即便在极高的温度下, 金也不与氢、氧、氮、硫、碳等物质

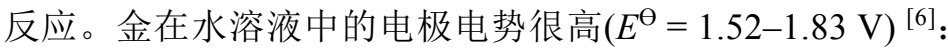

$$
\begin{array}{ll}
\mathrm{Au} \longrightarrow \mathrm{Au}^{+}+\mathrm{e}^{-} & E^{\Theta}=+1.83 \mathrm{~V} \\
\mathrm{Au} \longrightarrow \mathrm{Au}^{3+}+\mathrm{e}^{-} & E^{\Theta}=+1.52 \mathrm{~V}
\end{array}
$$

因此，在硝酸、硫酸、盐酸、氢氟酸以及碱中，金都不溶解。

而当金与配体形成配合物后, 能有效降低其电极电势。 $\mathrm{Au}^{+}$能与 $\mathrm{CN}^{-}$形成稳定的配合物, 并且电 极电势急剧降低至 $-0.596 \mathrm{~V}$ :

$$
4 \mathrm{Au}^{+}+8 \mathrm{CN}^{-}+\mathrm{O}_{2}+2 \mathrm{H}_{2} \mathrm{O}=4\left[\mathrm{Au}(\mathrm{CN})_{2}\right]^{-}+4 \mathrm{OH}^{-}
$$

由于 $E^{\Theta}\left(\left[\mathrm{Au}(\mathrm{CN})_{2}\right]^{-} / \mathrm{Au}\right)=-0.596 \mathrm{~V}<E^{\Theta}\left(\mathrm{O}_{2} / \mathrm{OH}^{-}\right)=0.401 \mathrm{~V}^{[6]}$, 故而溶金反应可以顺利进行, 这就是 利用配合物来溶金的原理。在王水法中, 盐酸实际上是起到了配体的作用, 经过硝酸氧化, 可以实 现溶金:

$\mathrm{Au}+\mathrm{HNO}_{3}+4 \mathrm{HCl}=\mathrm{HAuCl}_{4}+2 \mathrm{H}_{2} \mathrm{O}+\mathrm{NO} \uparrow$

在经过大量实验研究后, 我们发现, 合理选择其他配体, 可以实现溶金的效果, 这就为我们探 索绿色环保的溶金方法打开了思路。同时, 这些实验结果能帮助人们建立正确的观念, 起到良好的 作用。比如, 使用氯化钠也可以实现配体的作用, $\mathrm{Cl}^{-}$作为配体与金形成配合物后, 可以有效降低其 电极电势, 辅以醋酸提供质子, 在硝酸的氧化下, 可以使金溶解(醋酸的全面作用还须进一步研究)。 进而用维生素 $\mathrm{C}$ (抗坏血酸)将金还原，从而以更安全、更环保的方式实现固体废物资源化。

$$
\mathrm{Au}+\mathrm{HNO}_{3}+4 \mathrm{H}^{+}+4 \mathrm{Cl}^{-} \longrightarrow \mathrm{HAuCl}_{4}+2 \mathrm{H}_{2} \mathrm{O}+\mathrm{NO} \uparrow
$$

氯化钠和醋酸都是生活中常见的必需品, 在提高了安全性的同时也降低了腐蚀性。硝酸的加入 量, 不需僵化地采用一般教科书里王水中浓硝酸与浓盐酸体积比 $1: 3$ 或 $1: 4$ 的比例, 而采用以滴 至原料金恰好彻底溶解为标准, 这样既实现了对金的有效溶解, 又避免了溶液体系中多余硝酸对还 原步骤的影响。王水可以溶解原料中几乎所有金属, 造成溶液中多种离子对后期还原过程形成屏蔽 
干扰的弊端, 使用氯化钠后, 得到很大的改进。维生素 $\mathrm{C}$ 作为绿色安全的试剂, 还原结果令人满意。

2) 由于电子元器件种类繁多, 拓展运用配合物化学的原理, 进一步研究出对某些元器件更适合 的方法, 用退镀的形式来进行溶金。面对纷繁复杂的废旧元器件, 研究出多种有针对性的方法, 以 使得实践中对纷繁复杂的废旧元器件的处理更具有可行性。碘、碘化钾都是无毒的试剂, 同时金能 与 I-形成稳定的配合物, 金的碘配合物比金与溴、氯的配合物更加稳定, 应用碘化法可以从电子废 弃物中成功地浸取金 ${ }^{[7]}$ 。碘难溶于水, 但因与 $\mathrm{I}^{-}$生成 $\mathrm{I}_{3}^{-}$的缘故, 易溶于碘化物(如碘化钾)溶液中, 碘-碘 化钾溶液可以对金进行溶解, 从而实现对元器件的退镀。

$$
2 \mathrm{Au}+\mathrm{I}_{3}^{-}+\mathrm{I}^{-}=2 \mathrm{AuI}_{2}^{-}
$$

同时, 由于碘的氧化性在卤素中是最弱的，控制得法，还可以使基底金属不被腐蚀，从而有利 于基底元器件的再生使用。

对退镀液用亚硫酸钠还原, 可将金从溶液体系中还原出来。碘和碘类试剂价格昂贵, 将还原后 的溶液在酸性条件下用双氧水氧化，碘离子被氧化生成碘，可使溶剂中碘再生：

$$
2 \mathrm{I}^{-}+\mathrm{H}_{2} \mathrm{O}_{2}+2 \mathrm{H}^{+}=\mathrm{I}_{2}+2 \mathrm{H}_{2} \mathrm{O}
$$

这样, 在经济上更加合理, 又防止了生态环境的污染和处理废碘液带来的费用增加。

\section{2 试剂或材料}

科普演示实验涉及的试剂: 氯化钠、醋酸、 $\mathrm{V}_{\mathrm{C}}$ (抗坏血酸)、硝酸、碘化钾、碘、亚硫酸钠, 试 剂均为分析纯(AR)。

其他过程还涉及试剂: 硫酸、双氧水, 试剂均为分析纯(AR)。

\section{3 仪器和表征方法}

电感耦合等离子体光谱仪(Thermo 公司, 型号 ICAP6300)。

将熔炼后制得的金, 准确称取, 溶解于王水中, 稀释定容, 制成四氯合金酸溶液。由第三方专 业机构 “国土资源部贵阳矿产资源监督检测中心” 进行 ICP-AES 检测, 对其中铜、银、铁、镍、铅、 锰、锡、等元素进行分析测试。

\section{4 实验步骤/方法/现象}

原料预处理: 将废弃的电脑 CPU、内存条等含有金的电子元器件进行原料的预处理, 元器件分

拣、分离后, 将待处理的原料置于浓度为 $10 \%-20 \%$ 的硝酸溶液中, 固液质量比 $1: 15-1: 20$, 加热 至 $80^{\circ} \mathrm{C}$, 边加热边搅拌, 使元器件镀层内部金属与硝酸反应, 表面黄金成片状松动脱落。反应 8-10 h 后, 去除铜、镍等基体金属, 过滤, 将滤渣反复用去离子水清洗, 烘干备用。烘干后的滤渣 为富集度较高的原料备用, 可用于教学与科普实验。废水经环保处理。

\section{实验 1 用氯化钠溶液溶金并还原}

1) 硝酸按 1:1 比例与去离子水混合, 备用。在 $500 \mathrm{~mL}$ 烧杯中, 加入 $150 \mathrm{~mL}$ 去离子水, 加入 氯化钠 $34.0 \mathrm{~g}$ 、醋酸 $20 \mathrm{~mL}$, 放入原料金 $1.0 \mathrm{~g}$, 加热至沸腾, 冷却至 $80^{\circ} \mathrm{C}$ 后, 用滴管逐滴加入体 积比 $1: 1$ 的硝酸溶液, 边滴加边搅拌。溶液逐渐出现黄色, 颜色逐渐由浅变深, 继续滴加硝酸, 直 至原料金全部溶解为止。

2) 将溶金溶液冷却至室温, 用布什漏斗抽滤, 将滤液倒入烧杯中。滤液为橙黄色液体。

3) 加热赶硝。加入 1-2 粒沸石, 加热溶液至沸腾, 小火保持 $20 \mathrm{~min}$, 赶硝; 含金液挥发浓缩, 赶硝后, 补加去离子水, 恢复原有体积。

4) 在 $80^{\circ} \mathrm{C}$ 时, 逐量加入 $V_{C}$ 固体, 直至橙黄色彻底消失, 再加入 $5 \mathrm{~mL}$ 饱和 $V_{C}$ 溶液, 以使得 还原彻底。烧杯中出现浑浊, 静置 $1 \mathrm{~h}$, 烧杯底部沉淀出棕红色粉末, 即为粗金粉。

5) 将还原液静置, 沉淀, 取适量上清液, 滴入酸性 $\mathrm{SnCl}_{2}$ 溶液, 检验还原是否完全。没有出现 紫红色，表明金已彻底还原，此时残液中金的含量小于 $0.3 \mu \mathrm{g} \cdot \mathrm{mL}^{-1}$ 。

注: 从废弃物到熔炼金块的整个过程耗时较长, 可取涉及化学原理的 1)-5)步骤作为科普演示实 验。对公众关心的最终的 “熔炼金块” , 在此也完整介绍如下: 
6) 将彻底还原后的溶液用布什漏斗过滤, 用去离子水洗涤 4 次, 洗净滤渣, 并去除卤素离子。 取下滤纸上的粗金粉, 放入 $50 \mathrm{~mL}$ 烧杯中, 加入 $20 \mathrm{~mL}$ 稀硝酸, 加热, 搅拌, 直至沸腾。经过硝酸 处理，可溶解去除粗金粉中残余的其他金属。过滤，即得到金粉。

7) 将金粉放入坩埚中, 按质量比 $1: 1$ 的比例加入嗍砂, 硼砂可作为助熔剂, 并通过喼砂珠反 应进一步去除微量杂质。在马弗炉中逐级升温至 $1100^{\circ} \mathrm{C}$, 保温 $10 \mathrm{~min}$, 熔炼。冷却后, 取出, 将覆 盖有硼砂的金粒用稀硝酸处理，去掉表层嗍砂，得到具有美丽光泽的单质金。

\section{实验 2 用碘-碘化钾退镀元器件上的金镀层}

1) 在 $500 \mathrm{~mL}$ 烧杯中, 将 $34.0 \mathrm{~g}$ 碘化钾溶于 $150 \mathrm{~mL}$ 去离子水中, 加入 $11.0 \mathrm{~g}$ 碘, 搅拌溶解, 制成碘-碘化钾退镀溶液。

2) 将一片旧电脑 CPU 用铒子钳住, 置入溶液中, 浸没, $1 \mathrm{~min}$ 后可观察到金镀层出现溶解, 浸 没 $3 \mathrm{~min}$, 直至金镀层彻底溶解。取出元器件, 用洗瓶吹出去离子水洗涤, 洗下来的水收集在另一个 烧杯中。

3) 逐片继续将 CPU 浸入退镀液中, 重复 2)中的步骤, 完成 20 片 CPU 的退镀操作。

4) 以饱和的亚硫酸钠溶液作为还原液。将退镀液和洗液收集在一起, 往其中滴加还原液, 棕红 色的退镀液开始褪色, 继续滴加入还原液, 直至溶液棕红色彻底裉去后, 再加入 $3 \mathrm{~mL}$ 还原液, 以使 还原彻底。静置 $1 \mathrm{~h}$, 在烧杯底部沉积出棕红色粉末，即为粗金粉。

5) 将还原后的溶液静置, 沉淀, 取适量上清液, 滴入酸性 $\mathrm{SnCl}_{2}$ 溶液, 检验还原是否完全。没 有出现紫红色, 表明金已彻底还原, 此时残液中金的含量小于 $0.3 \mu \mathrm{g} \cdot \mathrm{mL}^{-1}$ 。

6) 重复实验 1 中 6)、7)两步过程, 可以得到具有美丽光泽的单质金。

注：从废弃物到熔炼金块的整个过程较长，我们可取 1)-5)的步骤作为科普演示实验。

还原液的再生: 在还原后的退镀液中, 滴加 $3 \mathrm{~mL}$ 硫酸, 加入 $7.0 \mathrm{~mL}$ 双氧水(AR、质量浓度 $30 \%$ ), 使得 $\mathrm{I}^{-}$氧化, 碘得以再生, 再生后的退镀液可以继续循环使用。

对退镀液环保性的检验: 金鱼是一种对生存环境要求苛刻的动物, 将退镀液每周都滴加入金鱼 缸中, 按每 $1 \mathrm{~L}$ 水中加入 $1 \mathrm{~mL}$ 退镀液的比例, 加入退镀液持续 6 个月以上, 娇气的金鱼可以依旧健 康地生活。

注：以上所有实验废水均收集后经环保处理。

分析检测：取实验 1 熔炼后制得的金, 准确称量 $0.1215 \mathrm{~g}$, 溶解于王水中, 稀释定容至 $100 \mathrm{~mL}$ 。 经 “国土资源部贵阳矿产资源监督检测中心” 进行 ICP-AES 检测, 对以下元素进行分析, 结果如 表 1 所示。扣除杂质含量后金块纯度 $>99.92 \%$ 。

表 1 ICP-AES 检测结果

\begin{tabular}{cc||cc}
\hline 元素 & 含量 $/\left(\mathrm{mg} \cdot \mathrm{L}^{-1}\right)$ & 元素 & 含量 $/\left(\mathrm{mg} \cdot \mathrm{L}^{-1}\right)$ \\
\hline $\mathrm{Ag}$ & 0.688 & $\mathrm{~Pb}$ & 0.059 \\
$\mathrm{Bi}$ & $<0.001$ & $\mathrm{Pd}$ & $<0.001$ \\
$\mathrm{Cd}$ & $<0.001$ & $\mathrm{Pt}$ & $<0.001$ \\
$\mathrm{Co}$ & $<0.001$ & $\mathrm{Rh}$ & $<0.001$ \\
$\mathrm{Cu}$ & 0.024 & $\mathrm{Ru}$ & $<0.001$ \\
$\mathrm{Fe}$ & $<0.003$ & $\mathrm{Se}$ & 0.005 \\
$\mathrm{Ir}$ & $<0.001$ & $\mathrm{Sn}$ & 0.061 \\
$\mathrm{Mn}$ & $<0.001$ & $\mathrm{Ti}$ & $<0.003$ \\
$\mathrm{Ni}$ & 0.062 & $\mathrm{Zn}$ & $<0.001$ \\
\hline
\end{tabular}




\section{3 科普展示和互动方案}

本实验中应用氯化钠溶液溶金、碘系退镀液溶金、还原等环节可通过演示实验的方式达到普及 科学原理的目的。本文旨在普及科学原理，不提倡公众因好奇与逐利心态从事 “淘金”。

科普环节适合的人群：对人群应有适当限制; 有关从业人员。

科普环节适合的地点：实验室、有隔离的展示台。

展示的内容：1）氯化钠溶液溶金；2) 退镀液对元器件的退镀；3) 还原制得金粉。

展示的形式: 1) 观摩操作；2) 互动。

互动的环节: 由专业人员准备好之后, 抽选身体健康、反应正常的观众来进行互动演示: 1) 由 观众上台, 检查溶液是不是家里每天都用的氯化钠盐, 检查是不是常见的醋酸, 激发观众兴趣; 2) 由观众滴加稀释过的硝酸, 直至原料中的金溶解; 3) 由观众持铌子将元器件浸入退镀液中, 现场 感受到金的溶解；4）由观众将退镀液滴加入金鱼缸中，检验试剂的环保性。

由专业人员介绍整体实验与原理，对环保溶金的环节进行展示。

\section{4 结语}

金, 是古往今来人们熟知的贵金属元素，“真金不怕火炼” 的古浐又使得金的特殊化学稳定性 家喻户晓, 社会公众对此广泛有着兴趣与好奇。随着手机、电脑等电子产品深入到全社会生活的方 方面面, 人们对电子元器件已是耳熟能详, 而氯化钠、醋酸是生活中人人皆知的必需品, 碘、碘化 钾是无毒试剂, 选择从社会公众熟悉的事物入手, 容易使得科学原理在通俗中引入, 并合理引导公 众的好奇心态，易使得公众在通俗中理解枯燥的化学原理，从而展现化学之美。

不少公众对王水溶金和氧化物溶金有所耳闻, 但由于一知半解, 还有不少误区。普及科学知识, 从而树立科学理念, 避免好奇心趋势下带来的危险与危害。同时, 面对日益增多的电子废弃物, 也 需要引导公众树立环保与回收的观念, 认识电子垃圾, 崇尚垃圾分类。

对化学化工类的学生而言, 本实验可以进一步认识配合物, 学习化学基本原理, 激发环保、创 新的科学思路。让学生在好奇中体会化学这门古老而又永远充满生命力的学科, 经典的原理在任何 时代都是文明进步的利器。

\section{5 特点/特色/创新性声明}

本科普实验针对目前突出的环境问题, 结合公众的广泛好奇, 巧妙利用配合物化学的原理, 寓 教于乐。其中原创实验方法已获国家发明专利受理(专利名称：一种绿色溶解并提取金元素的方法, 受理号: 201911008127)。

特色：1) 及时针对社会突出问题；2) 推动垃圾分类、资源回收；3) 绿色环保。

特点：1）杜绝高危试剂, 选用安全试剂；2) 提取率高、产品纯度高；3) 寓教于乐。

特别声明: 本作品旨在普及科学原理, 提高公众素质, 并帮助大学生进一步认识配合物性质; 电子废弃物的回收利用须由具备有关资质的企业进行。

\section{参 考 文 献}

[1] 吴骏, 陈亮, 邱丽娟. 黄金, 2008, $29(6), 55$.

[2] 徐秀丽, 李晶荣. 青岛大学学报, 2011, 26 (2), 69.

[3] 朱经发, 林辉东, 王德汉. 土壤与环境, 2002, 11 (3), 307.

[4] 联合国报告称中国已成全球最大电子“垃圾场”. 人民网. [2013-06-12]. http://finance.people.com.cn/n/2013/0612/c70846-21818262.html.

[5] 报告称世界 70\%电子产品最终变成垃圾流向中国. 新浪网. [2013-06-10]. https://finance.sina.com.cn/chanjing/cyxw/20130610/102615760366.shtml.

[6] 杨宏孝, 天津大学无机化学教研室. 无机化学. 北京: 高等教育出版社, 2002: 428-437.

[7] 徐渠, 陈东辉, 陈亮, 黄满红. 有色金属, 2010, 62 (3), 88. 